\title{
Agronomic Fortification with Zinc and Iron to Enhancing Micronutrient Concentration in Sweet corn grain to ameliorate the Deficiency Symptoms in Human Beings
}

\author{
Fakeerappa Arabhanvi* and U.K. Hulihalli
}

\begin{abstract}
Department of Agronomy, College of Agriculture, University of Agricultural Sciences, Dharwad-580 005, Karnataka, India

*Corresponding author
\end{abstract}

A B S T R A C T

Keywords

Agronomic,

Fortification,

Micronutrients,

Sweet corn

Article Info

Accepted:

04 January 2018

Available Online:

10 February 2018
A field experiment was conducted at Main Agricultural Research Station, Dharwad, to study the agronomic fortification with Zinc and Iron to enhancing micronutrient concentration in sweet corn during Kharif 2015.-16 and 2016-17. The field experiment was laid out in split plot design with three replications and 21 treatment combinations involving three main and seven subplots. The pooled results indicated that, soil application of $\mathrm{ZnSO}_{4}$ and $\mathrm{FeSO}_{4}\left(10 \mathrm{~kg}\right.$ each ha $\left.{ }^{-1}\right)$ fortified with vermicompost $\left(250 \mathrm{~kg} \mathrm{ha}^{-1}\right)$ along with foliar application of $\mathrm{ZnSO}_{4}$ and $\mathrm{FeSO}_{4}(1.0 \%$ each) at 20 and 40 days after sowing were recorded significantly higher zinc and iron concentration in grain and fodder during both seasons, which was on par with treatment combination of soil application of $\mathrm{ZnSO}_{4}$ and $\mathrm{FeSO}_{4}\left(10 \mathrm{~kg}\right.$ each ha $\left.{ }^{-1}\right)$ fortified with farm yard manure $\left(500 \mathrm{~kg} \mathrm{ha}^{-1}\right)$ along with foliar application of $\mathrm{ZnSO}_{4}$ and $\mathrm{FeSO}_{4}(1.0 \%$ each) at 20 and 40 days. Similarly, significantly higher micronutrients uptake was also observed in above said treatments as compared to no fortification and no foliar application of micronutrients.

\section{Introduction}

Specialty corns (viz., sweet corn, popcorn, baby corn and high oil corn) assume tremendous market potential not only in India but also in the international market. These specialty corns with their high market value are perfectly suitable to peri-urban agriculture. Thus they promise higher income to maize growers. Out of the various specialty corns, sweet corn (Zea mays L. var. saccharata Sturt) has a big market potential. It is a hybridized variety of maize specifically bred to increase the sugar content. Large population of human beings in developing countries is mainly reliant on a staple diet of cereals, such as rice, wheat and maize. Unfortunately all of our major cereal food crops lack certain essential vitamins and minerals, as milled cereal grains are poor sources of lysine, vitamin A, folic acid, iron, zinc and selenium, which are essential for normal growth and metabolism of human beings. In Asia about 35 per cent of children between age group of 0 and 5 years suffer from zinc or irondeficiencies, 250 million people suffer from vitamin A deficiency and 58 per cent of pregnant women in developing countries are 
anemic from iron deficiency (Cababallero, 2002). As per the World Health Organization (Anon., 2000) the daily dietary should have zinc intake of (infants for $5 \mathrm{mg} \mathrm{day}^{-1}$, children $10 \mathrm{mg}$ day $^{-1}$, women $12 \mathrm{mg}$ day $^{-1}$, pregnant women $15 \mathrm{mg} \mathrm{day}^{-1}$, lactating women $16 \mathrm{mg}$ day $^{-1}$ and men $15 \mathrm{mg} \mathrm{day}^{-1}$ ) and iron intake of (birth to 6 months $0.27 \mathrm{mg} \mathrm{day}^{-1}, 1-3$ year 7.0 $\mathrm{mg} \mathrm{day}^{-1}, 4-8$ years $8 \mathrm{mg}$ day $^{-1}, 9-51$ year 10 $\mathrm{mg}$ day $^{-1}$ and pregnant women $27 \mathrm{mg} \mathrm{day}^{-1}$ ). Intake of zinc and iron less than quantity specified by WHO may leads to slow down the physiological processes because of zinc deficiency retarded the growth, skeletal abnormalities, delayed wound healing, increased abortion risk and diarrhea (Salgueiro et al., 2000). Iron deficiency induced anemia is most widespread micronutrient deficiency in human beings and it results in impaired physical growth, mental development and learning capacity (Bouis, 2003). These micronutrients deficiencies in soil are not only hampering the crop productivity but also in deteriorating the quality produce.

Biofortification is the process of creating micronutrient denser staple food crops with increased bioavailable concentrations through agronomic intervention or genetic selection (Cakmak, 2008). Biofortification works for twin objective of increasing the concentration of the micronutrients in the grains and simultaneously improving the bioavailability of micronutrients in the grains to alleviate the micronutrient deficiency in human beings and also animals. It is a potential cost-effective and sustainable agronomic way to enrich the micronutrient content of food grains. It is an upcoming strategy for dealing the deficiencies of micronutrients in the developing world. World Health Organization (Anon., 2000) has estimated that biofortification of iron could help in curing two billion people suffering from iron deficiency-induced anemia. Agronomic fortification strategy appears to be essential in keeping sufficient amount of available zinc and iron in soil solution and maintaining adequate zinc and iron transport to the grains during reproductive stage. It is very attractive and useful strategy in solving zinc and iron deficiency related health problems globally and effectively. Hence, In view of these facts, present investigation on "Agronomic fortification with Zinc and Iron to enhancing micronutrient concentration in sweet corn grain to ameliorate the deficiency symptoms in human beings".

\section{Materials and Methods}

The field experiment was conducted at University of Agricultural Sciences, Dharwad of Northern Transition Zone of Karnataka, during kharif 2015-16 and 2016-17. The field experiment was laid out in Split plot design with three replications. There were 21 treatment combinations involving three main plots (soil applications) viz., $\mathrm{S}_{1}-\mathrm{ZnSO}_{4} \&$ $\mathrm{FeSO}_{4} @ 10 \mathrm{~kg}$ each ha ${ }^{-1}, \mathrm{~S}_{2}-\mathrm{ZnSO}_{4} \& \mathrm{FeSO}_{4}$ @ $10 \mathrm{~kg} \mathrm{each} \mathrm{ha}^{-1}$ (Fortified with $\mathrm{VC}$ at 250 $\mathrm{kg}$ ) and $\mathrm{S}_{3}-\mathrm{ZnSO}_{4} \& \mathrm{FeSO}_{4} @ 10 \mathrm{~kg}$ each ha ${ }^{-1}$ (fortified with FYM at $500 \mathrm{~kg}$ ) and seven subplots (foliar applications) viz., $\left(\mathrm{F}_{1}-\mathrm{ZnSO}_{4}\right.$ \& $\mathrm{FeSO}_{4} @ 0.5 \%$ each at $20 \mathrm{DAS}, \mathrm{F}_{2}-\mathrm{ZnSO}_{4}$ \& $\mathrm{FeSO}_{4} @ 0.5 \%$ each at $40 \mathrm{DAS}, \mathrm{F}_{3^{-}} \mathrm{ZnSO}_{4}$ $\& \mathrm{FeSO}_{4} @ 0.5 \%$ each at 20 and $40 \mathrm{DAS}, \mathrm{F}_{4}-$ $\mathrm{ZnSO}_{4} \& \mathrm{FeSO}_{4} @ 1.0 \%$ each at $20 \mathrm{DAS}, \mathrm{F}_{5}-$ $\mathrm{ZnSO}_{4} \& \mathrm{FeSO}_{4} @ 1.0 \%$ each at 40 DAS, F $6^{-}$ $\mathrm{ZnSO}_{4} \& \mathrm{FeSO}_{4} @ 1.0 \%$ each at 20 and 40 DAS, $F_{7}$ - Control (No foliar spray). Note: Recommended dose of 100:50:25 N: $\mathrm{P}_{2} \mathrm{O}_{5}$ : $\mathrm{K}_{2} \mathrm{O} \mathrm{kg} \mathrm{ha}{ }^{-1}, 50 \%$ of $\mathrm{N}$ applied at basal, $25 \%$ at 30 DAS and remaining $25 \%$ appliéd at 45 DAS. Full dose of $\mathrm{P}_{2} \mathrm{O}_{5}$ and $\mathrm{K}_{2} \mathrm{O}$ were applied at the time of sowing. Enrichment procédure: Zinc sulphate and iron sulphate was thoroughly mixed with farm yard manure and vermicompost as per the treatments. The mixture was covered with polythene sheet for natural process of composting for 45 days to fix the externally added inorganic $\mathrm{Zn}$ and $\mathrm{Fe}$ into organically bound and naturally chelated 
form of $\mathrm{Zn}$ and $\mathrm{Fe}$ and maintaining 50 per cent moisture in enriched compost. These enriched manures were utilized as a soil application at the time of sowing of sweetcorn. The test crop was sweetcorn (Hybrid sugar 75). Zinc and iron, content were determined in an aliquot of the diacid digested extract by using Atomic Absorption Spectrophotometer (AAS) method as described by Follett and Lindsay (1969).

Nutrient uptake $\left(\mathrm{g} \mathrm{ha}^{-1}\right)=($ Nutrient content $(\%) / 1000) \times$ Dry matter yield $\left(\mathrm{kg} \mathrm{ha}^{-1}\right)$.

The mean value of sub sub plot and interaction were separately subjected to Duncan's multiple range test (DMRT) using the corresponding error mean sum of squares and degrees of freedom values.

\section{Results and Discussion}

Micronutrient concentration in grain and fodder

Individual years as well as pooled data are presented in Table 1. Zinc concentration of grain and fodder in sweet corn differed significantly due to different treatment combinations.

Pooled results indicated that, soil application of $\mathrm{ZnSO}_{4}$ and $\mathrm{FeSO}_{4}\left(10 \mathrm{~kg}\right.$ each ha $\left.{ }^{-1}\right)$ fortified with vermicompost $\left(250 \mathrm{~kg} \mathrm{ha}^{-1}\right)$ along with foliar application of $\mathrm{ZnSO}_{4}$ and $\mathrm{FeSO}_{4}(1.0 \%)$ each at 20 and 40 DAS $\left(\mathrm{S}_{2} \mathrm{~F}_{6}\right)$ was recorded significantly higher zinc concentration (35.98 and $47.29 \mathrm{ppm}$ ) in grain and fodder, respectively, which was on par with treatment combinations of $\mathrm{S}_{3} \mathrm{~F}_{6}, \quad \mathrm{~S}_{2} \mathrm{~F}_{3}$ and $\mathrm{S}_{1} \mathrm{~F}_{6}$ as compared to $\mathrm{S}_{1} \mathrm{~F}_{7}$ (26.33 and $34.93 \mathrm{ppm}$, respectively, in grain and fodder) (Table 1). Similarly, iron concentration of sweet corn grain and fodder differed significantly due to different treatment combinations. Soil application of $\mathrm{ZnSO}_{4}$ and $\mathrm{FeSO}_{4}(10 \mathrm{~kg}$ each $\left.\mathrm{ha}^{-1}\right)$ fortified with vermicompost $\left(250 \mathrm{~kg} \mathrm{ha}^{-1}\right)$ along with foliar application of $\mathrm{ZnSO}_{4}$ and $\mathrm{FeSO}_{4}(1.0 \%)$ each at 20 and 40 DAS $\left(\mathrm{S}_{2} \mathrm{~F}_{6}\right)$ was recorded significantly higher iron concentration (109.41 and $202.91 \mathrm{ppm}$ ) in grain and fodder which was on par with treatment combinations of $\mathrm{S}_{3} \mathrm{~F}_{6}, \mathrm{~S}_{2} \mathrm{~F}_{3}$ and $\mathrm{S}_{1} \mathrm{~F}_{6}$ as compared to $\mathrm{S}_{1} \mathrm{~F}_{7}$ (Table 2). The higher micronutrient concentration in grain and fodder was due to fortified vermicompost with zinc sulphate and iron sulphate which improved the micronutrient concentration in the soil solution that facilitated greater absorption and translocation from source to sink. The similar results were obtained by Nayak and Panda (2000) and Habib (2009).

Uptake of micronutrients by grain and fodder

Pooled results indicated that, significantly higher zinc uptake (232.39 and $493.66 \mathrm{~g} \mathrm{ha}^{-1}$ ) in grain and fodder was recorded with treatment combination of soil application of $\mathrm{ZnSO}_{4}$ and $\mathrm{FeSO}_{4}\left(10 \mathrm{~kg}\right.$ each ha $\left.{ }^{-1}\right)$ fortified with vermicompost $\left(250 \mathrm{~kg} \mathrm{ha}^{-1}\right)$ along with foliar application of $\mathrm{ZnSO}_{4}$ and $\mathrm{FeSO}_{4}(1.0 \%)$ each at 20 and 40 DAS $\left(\mathrm{S}_{2} \mathrm{~F}_{6}\right)$. Whereas, significantly lower zinc uptake was recorded with treatment combination of $\mathrm{S}_{1} \mathrm{~F}_{7}$ (Table 3 ).

Similarly, iron uptake significantly higher (715.46 and 2201.46 $\mathrm{g} \mathrm{ha}^{-1}$ ) in grain and fodder was recorded with soil application of $\mathrm{ZnSO}_{4}$ and $\mathrm{FeSO}_{4}\left(10 \mathrm{~kg}\right.$ each ha $\left.{ }^{-1}\right)$ fortified with vermicompost $\left(250 \mathrm{~kg} \mathrm{ha}^{-1}\right)$ along with foliar application of $\mathrm{ZnSO}_{4}$ and $\mathrm{FeSO}_{4}(1.0 \%)$ each at 20 and 40 DAS $\left(\mathrm{S}_{2} \mathrm{~F}_{6}\right)$ (Table 4). Whereas, significantly lower iron uptake was recorded with treatment combination of $\mathrm{S}_{1} \mathrm{~F}_{7}$. Higher uptake of micronutrients it might be due to microbial decomposition of zinc and iron fortified vermicompost and farm yard manure with simultaneous release of organic acids might have favored the availability of micro nutrients in soil and their uptake by sweet corn. 
Table.1 Zinc concentration in grain and fodder of sweet corn as influenced by agronomic fortification with zinc and iron

\begin{tabular}{|c|c|c|c|c|c|c|}
\hline \multirow[t]{2}{*}{ Treatments } & \multicolumn{3}{|c|}{ Zinc concentration in grain (ppm) } & \multicolumn{3}{|c|}{ Zinc concentration in fodder (ppm) } \\
\hline & 2015-16 & 2016-17 & Pooled & 2015-16 & 2016-17 & Pooled \\
\hline \multicolumn{7}{|l|}{ Main plot - Soil application } \\
\hline $\mathrm{S}_{1}-\mathrm{ZnSO}_{4} \& \mathrm{FeSO}_{4} @ 10 \mathrm{~kg}$ each ha ${ }^{-1}$ (No fortification) & $30.86 \mathrm{~b}$ & $30.90 \mathrm{~b}$ & $30.88 \mathrm{~b}$ & $41.10 \mathrm{~b}$ & $41.21 \mathrm{~b}$ & $41.15 b$ \\
\hline $\mathrm{S}_{2}-\mathrm{ZnSO}_{4} \& \mathrm{FeSO}_{4} @ 10 \mathrm{~kg} \mathrm{each} \mathrm{ha}^{-1}$ (Fortified with VC at $250 \mathrm{~kg}$ ) & $31.89 \mathrm{a}$ & $31.96 \mathrm{a}$ & $31.93 \mathrm{a}$ & $42.52 \mathrm{a}$ & $42.82 \mathrm{a}$ & 42.67 a \\
\hline $\mathrm{S}_{3}-\mathrm{ZnSO}_{4} \& \mathrm{FeSO}_{4} @ 10 \mathrm{~kg}$ each ha ${ }^{-1}$ (Fortified with FYM at $500 \mathrm{~kg}$ ) & $31.68 \mathrm{a}$ & $31.76 \mathrm{a}$ & $31.72 \mathrm{a}$ & $42.15 \mathrm{ab}$ & $42.23 \mathrm{ab}$ & $42.19 \mathrm{ab}$ \\
\hline S.Em. \pm & 0.10 & 0.13 & 0.10 & 0.34 & 0.32 & 0.31 \\
\hline \multicolumn{7}{|l|}{ Sub-plot - Foliar application } \\
\hline $\mathrm{F}_{1}-\mathrm{ZnSO}_{4} \& \mathrm{FeSO}_{4} @ 0.5 \%$ each at $20 \mathrm{DAS}$ & $29.29 \mathrm{c}$ & $29.41 \mathrm{c}$ & $29.35 \mathrm{c}$ & $38.95 \mathrm{c}$ & $39.16 \mathrm{~d}$ & $39.06 \mathrm{~d}$ \\
\hline $\mathrm{F}_{2}-\mathrm{ZnSO}_{4} \& \mathrm{FeSO}_{4} @ 0.5 \%$ each at $40 \mathrm{DAS}$ & $30.53 \mathrm{c}$ & $30.57 \mathrm{c}$ & $30.55 \mathrm{c}$ & $42.11 \mathrm{~b}$ & $42.31 \mathrm{c}$ & $42.21 \mathrm{c}$ \\
\hline $\mathrm{F}_{3}-\mathrm{ZnSO}_{4} \& \mathrm{FeSO}_{4} @ 0.5 \%$ each at 20 and $40 \mathrm{DAS}$ & $34.28 \mathrm{ab}$ & $34.39 \mathrm{ab}$ & $34.33 \mathrm{ab}$ & $45.41 \mathrm{a}$ & $45.41 \mathrm{ab}$ & $45.41 \mathrm{ab}$ \\
\hline $\mathrm{F}_{4}-\mathrm{ZnSO}_{4} \& \mathrm{FeSO}_{4} @ 1.0 \%$ each at $20 \mathrm{DAS}$ & $30.51 \mathrm{c}$ & $30.66 \mathrm{c}$ & $30.58 \mathrm{c}$ & $40.71 \mathrm{bc}$ & $40.92 \mathrm{c}$ & $40.82 \mathrm{~cd}$ \\
\hline $\mathrm{F}_{5}-\mathrm{ZnSO}_{4} \& \mathrm{FeSO}_{4} @ 1.0 \%$ each at $40 \mathrm{DAS}$ & $33.25 \mathrm{~b}$ & $33.29 \mathrm{~b}$ & $33.27 \mathrm{~b}$ & $44.36 \mathrm{a}$ & $44.57 \mathrm{~b}$ & $44.47 \mathrm{~b}$ \\
\hline $\mathrm{F}_{6}-\mathrm{ZnSO}_{4} \& \mathrm{FeSO}_{4} @ 1.0 \%$ each at 20 and $40 \mathrm{DAS}$ & $35.28 \mathrm{a}$ & $35.34 \mathrm{a}$ & $35.31 \mathrm{a}$ & $46.55 \mathrm{a}$ & $46.72 \mathrm{a}$ & $46.64 \mathrm{a}$ \\
\hline $\mathrm{F}_{7}$ - Control (No foliar spray) & $27.21 \mathrm{~d}$ & $27.13 \mathrm{~d}$ & $27.17 \mathrm{~d}$ & $35.38 \mathrm{~d}$ & $35.51 \mathrm{e}$ & $35.44 \mathrm{e}$ \\
\hline S.Em. \pm & 0.52 & 0.49 & 0.50 & 0.75 & 0.60 & 0.64 \\
\hline \multicolumn{7}{|l|}{ Interaction } \\
\hline $\mathrm{S}_{1} \mathrm{~F}_{1}$ & $29.50 \mathrm{~d}-\mathrm{g}$ & $29.57 \mathrm{~d}-\mathrm{g}$ & $29.53 \mathrm{~d}-\mathrm{g}$ & $38.79 \mathrm{~g}$ & $38.93 \mathrm{hi}$ & $38.86 \mathrm{gh}$ \\
\hline $\mathbf{S}_{1} \mathrm{~F}_{2}$ & $30.13 \mathrm{~d}-\mathrm{g}$ & $30.27 \mathrm{~d}-\mathrm{g}$ & $30.20 \mathrm{~d}-\mathrm{g}$ & $41.74 \mathrm{~d}-\mathrm{f}$ & $41.83 \mathrm{e}-\mathrm{h}$ & $41.79 \mathrm{~d}-\mathrm{g}$ \\
\hline $\mathrm{S}_{1} \mathrm{~F}_{3}$ & $32.23 \mathrm{~b}-\mathrm{d}$ & $32.40 \mathrm{~b}-\mathrm{d}$ & $32.32 \mathrm{~b}-\mathrm{d}$ & $43.33 \mathrm{~b}-\mathrm{d}$ & $43.37 \mathrm{~b}-\mathrm{f}$ & $43.35 \mathrm{~b}-\mathrm{e}$ \\
\hline $\mathbf{S}_{1} \mathbf{F}_{4}$ & $31.03 \mathrm{c}-\mathrm{e}$ & $31.07 \mathrm{c}-\mathrm{e}$ & 31.05 c-e & $39.90 \mathrm{e}-\mathrm{g}$ & $40.05 \mathrm{f}-\mathrm{h}$ & $39.98 \mathrm{e}-\mathrm{g}$ \\
\hline $\mathrm{S}_{1} \mathrm{~F}_{5}$ & $32.20 \mathrm{~b}-\mathrm{d}$ & $32.30 \mathrm{~b}-\mathrm{d}$ & $32.25 \mathrm{~b}-\mathrm{d}$ & $43.30 \mathrm{~b}-\mathrm{d}$ & 43.45 b-f & $43.38 \mathrm{~b}-\mathrm{e}$ \\
\hline $\mathrm{S}_{1} \mathrm{~F}_{6}$ & $34.50 \mathrm{ab}$ & $34.50 \mathrm{ab}$ & $34.50 \mathrm{ab}$ & $45.96 \mathrm{ab}$ & $45.61 \mathrm{a}-\mathrm{d}$ & $45.78 \mathrm{a}-\mathrm{c}$ \\
\hline $\mathbf{S}_{1} \mathbf{F}_{7}$ & $26.43 \mathrm{~h}$ & $26.23 \mathrm{~h}$ & $26.33 \mathrm{~h}$ & $34.67 \mathrm{~h}$ & $35.20 \mathrm{j}$ & $34.93 \mathrm{i}$ \\
\hline $\mathbf{S}_{2} \mathbf{F}_{1}$ & $29.13 \mathrm{e}-\mathrm{h}$ & $29.33 \mathrm{e}-\mathrm{g}$ & $29.23 \mathrm{e}-\mathrm{g}$ & $39.23 \mathrm{fg}$ & $39.54 \mathrm{gh}$ & $39.39 \mathrm{f}-\mathrm{h}$ \\
\hline $\mathbf{S}_{2} \mathrm{~F}_{2}$ & $30.96 \mathrm{c}-\mathrm{e}$ & $31.13 \mathrm{c}-\mathrm{e}$ & $31.05 \mathrm{c}-\mathrm{e}$ & $42.55 \mathrm{c}-\mathrm{e}$ & $42.86 \mathrm{c}-\mathrm{g}$ & $42.71 \mathrm{c}-\mathrm{f}$ \\
\hline $\mathrm{S}_{2} \mathrm{~F}_{3}$ & $35.53 \mathrm{a}$ & 35.57 a & $35.55 \mathrm{a}$ & $46.49 \mathrm{a}$ & $46.70 \mathrm{ab}$ & $46.60 \mathrm{ab}$ \\
\hline $\mathbf{S}_{2} \mathbf{F}_{4}$ & $30.20 \mathrm{~d}-\mathrm{g}$ & $30.43 \mathrm{~d}-\mathrm{f}$ & $30.32 \mathrm{~d}-\mathrm{g}$ & $41.40 \mathrm{~d}-\mathrm{g}$ & $41.71 \mathrm{e}-\mathrm{h}$ & $41.55 \mathrm{~d}-\mathrm{g}$ \\
\hline $\mathrm{S}_{2} \mathbf{F}_{5}$ & $34.21 \mathrm{ab}$ & $34.21 \mathrm{ab}$ & $34.21 \mathrm{ab}$ & $45.00 \mathrm{a}-\mathrm{c}$ & $45.31 \mathrm{a}-\mathrm{d}$ & $45.15 \mathrm{a}-\mathrm{d}$ \\
\hline $\mathrm{S}_{2} \mathrm{~F}_{6}$ & $35.50 \mathrm{a}$ & $35.40 \mathrm{a}$ & $35.98 \mathrm{a}$ & $47.09 \mathrm{a}$ & $47.50 \mathrm{a}$ & $47.29 \mathrm{a}$ \\
\hline $\mathbf{S}_{2} \mathbf{F}_{7}$ & $27.70 \mathrm{f}-\mathrm{h}$ & $27.67 \mathrm{f}-\mathrm{h}$ & $27.68 \mathrm{f}-\mathrm{h}$ & $35.87 \mathrm{~h}$ & $36.14 \mathrm{ij}$ & $36.00 \mathrm{hi}$ \\
\hline $\mathrm{S}_{3} \mathrm{~F}_{1}$ & $29.23 \mathrm{~d}-\mathrm{h}$ & $29.33 \mathrm{e}-\mathrm{g}$ & $29.28 \mathrm{e}-\mathrm{g}$ & $38.83 \mathrm{~g}$ & $39.01 \mathrm{hi}$ & $38.92 \mathrm{gh}$ \\
\hline $\mathrm{S}_{3} \mathrm{~F}_{2}$ & $30.50 \mathrm{c}-\mathrm{f}$ & $30.30 \mathrm{~d}-\mathrm{g}$ & $30.40 \mathrm{~d}-\mathrm{f}$ & $42.04 \mathrm{de}$ & $42.22 \mathrm{~d}-\mathrm{h}$ & $42.13 \mathrm{~d}-\mathrm{g}$ \\
\hline $\mathrm{S}_{3} \mathrm{~F}_{3}$ & $35.07 \mathrm{ab}$ & $35.20 \mathrm{a}$ & $35.13 \mathrm{a}$ & $46.40 \mathrm{a}$ & $46.15 \mathrm{a}-\mathrm{c}$ & $46.28 \mathrm{a}-\mathrm{c}$ \\
\hline $\mathbf{S}_{3} \mathbf{F}_{4}$ & $30.30 \mathrm{~d}-\mathrm{g}$ & $30.47 \mathrm{~d}-\mathrm{f}$ & $30.38 \mathrm{~d}-\mathrm{f}$ & $40.83 \mathrm{~d}-\mathrm{e}$ & $41.01 \mathrm{f}-\mathrm{h}$ & $40.92 \mathrm{e}-\mathrm{g}$ \\
\hline $\mathbf{S}_{3} \mathbf{F}_{5}$ & $33.33 \mathrm{a}-\mathrm{c}$ & $33.37 \mathrm{a}-\mathrm{c}$ & $33.35 \mathrm{a}-\mathrm{c}$ & $44.78 \mathrm{a}-\mathrm{c}$ & 44.96 a-e & $44.87 \mathrm{a}-\mathrm{d}$ \\
\hline $\mathrm{S}_{3} \mathrm{~F}_{6}$ & $35.83 \mathrm{a}$ & $36.13 \mathrm{a}$ & $35.45 \mathrm{a}$ & $46.60 \mathrm{a}$ & 47.07 a-e & $46.83 \mathrm{ab}$ \\
\hline $\mathbf{S}_{3} \mathbf{F}_{7}$ & $27.50 \mathrm{gh}$ & $27.50 \mathrm{gh}$ & $27.50 \mathrm{gh}$ & $35.59 \mathrm{~h}$ & $35.19 \mathrm{j}$ & $35.39 \mathrm{i}$ \\
\hline S.Em. \pm & 0.89 & 0.857 & 0.86 & 0.85 & 1.03 & 1.10 \\
\hline
\end{tabular}

Means followed by the same letter (s) within a column are not significantly differed by DMRT $(\mathrm{P}=0.05)$

Initial grain Zn: $24.52 \mathrm{ppm}$ in 2015 and $23.40 \mathrm{ppm}$ in 2016 
Table.2 Iron concentration in grain and fodder of sweet corn as influenced by agronomic fortification with zinc and iron

\begin{tabular}{|c|c|c|c|c|c|c|}
\hline \multirow[t]{2}{*}{ Treatments } & \multicolumn{3}{|c|}{ Iron concentration in grain (ppm) } & \multicolumn{3}{|c|}{ Iron concentration in fodder (ppm) } \\
\hline & 2015-16 & 2016-17 & Pooled & 2015-16 & 2016-17 & Pooled \\
\hline \multicolumn{7}{|l|}{ Main plot - Soil application } \\
\hline $\mathrm{S}_{1}-\mathrm{ZnSO}_{4} \& \mathrm{FeSO}_{4} @ 10 \mathrm{~kg}$ each ha ${ }^{-1}$ (No fortification) & $102.70 \mathrm{a}$ & $102.68 \mathrm{~b}$ & $102.69 \mathrm{~b}$ & $201.29 \mathrm{~b}$ & $201.89 \mathrm{a}$ & $201.59 \mathrm{a}$ \\
\hline $\mathrm{S}_{2}-\mathrm{ZnSO}_{4} \& \mathrm{FeSO}_{4} @ 10 \mathrm{~kg} e a c h ~ h a^{-1}$ (Fortified with VC at $250 \mathrm{~kg}$ ) & $104.14 \mathrm{a}$ & $104.29 \mathrm{a}$ & $104.22 \mathrm{a}$ & 202.90 a & $202.92 \mathrm{a}$ & $202.91 \mathrm{a}$ \\
\hline $\mathrm{S}_{3}-\mathrm{ZnSO}_{4} \& \mathrm{FeSO}_{4} @ 10 \mathrm{~kg}$ each ha ${ }^{-1}$ (Fortified with FYM at $500 \mathrm{~kg}$ ) & $103.33 \mathrm{a}$ & $103.70 \mathrm{ab}$ & $103.51 \mathrm{ab}$ & $202.64 \mathrm{a}$ & $202.83 \mathrm{a}$ & 202.74 a \\
\hline S.Em. \pm & 0.45 & 0.26 & 0.32 & 0.33 & 0.34 & 0.33 \\
\hline \multicolumn{7}{|l|}{ Sub-plot - Foliar application } \\
\hline $\mathrm{F}_{1}-\mathrm{ZnSO}_{4} \& \mathrm{FeSO}_{4} @ 0.5 \%$ each at $20 \mathrm{DAS}$ & $100.71 \mathrm{c}$ & $100.89 \mathrm{c}$ & $100.80 \mathrm{~d}$ & $198.71 \mathrm{c}$ & $198.87 \mathrm{c}$ & $198.79 \mathrm{c}$ \\
\hline $\mathrm{F}_{2}-\mathrm{ZnSO}_{4} \& \mathrm{FeSO}_{4} @ 0.5 \%$ each at $40 \mathrm{DAS}$ & $103.65 \mathrm{bc}$ & $103.70 \mathrm{bc}$ & $103.68 \mathrm{bc}$ & $201.40 \mathrm{c}$ & $201.54 \mathrm{c}$ & $201.47 \mathrm{c}$ \\
\hline $\mathrm{F}_{3}-\mathrm{ZnSO}_{4} \& \mathrm{FeSO}_{4} @ 0.5 \%$ each at 20 and $40 \mathrm{DAS}$ & $107.30 \mathrm{a}$ & $106.61 \mathrm{ab}$ & $106.96 \mathrm{a}$ & $207.91 \mathrm{ab}$ & $208.03 \mathrm{ab}$ & $207.97 \mathrm{ab}$ \\
\hline $\mathrm{F}_{4}-\mathrm{ZnSO}_{4} \& \mathrm{FeSO}_{4} @ 1.0 \%$ each at $20 \mathrm{DAS}$ & $101.93 \mathrm{c}$ & $102.10 \mathrm{c}$ & $102.01 \mathrm{~cd}$ & $199.16 \mathrm{c}$ & $199.51 \mathrm{c}$ & $199.33 \mathrm{c}$ \\
\hline $\mathrm{F}_{5}-\mathrm{ZnSO}_{4} \& \mathrm{FeSO}_{4} @ 1.0 \%$ each at $40 \mathrm{DAS}$ & $106.12 \mathrm{ab}$ & $106.35 \mathrm{ab}$ & $106.23 \mathrm{ab}$ & $205.75 \mathrm{~b}$ & $205.94 \mathrm{~b}$ & $205.84 \mathrm{~b}$ \\
\hline $\mathrm{F}_{6}-\mathrm{ZnSO}_{4} \& \mathrm{FeSO}_{4} @ 1.0 \%$ each at 20 and $40 \mathrm{DAS}$ & $108.53 \mathrm{a}$ & 108.59 a & $108.56 \mathrm{a}$ & 209.88 a & 209.89 a & $209.88 \mathrm{a}$ \\
\hline $\mathbf{F}_{7}-$ Control (No foliar spray) & $95.49 \mathrm{~d}$ & $96.66 \mathrm{~d}$ & $96.08 \mathrm{e}$ & $193.15 \mathrm{~d}$ & $194.07 \mathrm{~d}$ & $193.61 \mathrm{~d}$ \\
\hline S.Em. \pm & 0.99 & 1.04 & 1.02 & 1.04 & 1.13 & 1.08 \\
\hline \multicolumn{7}{|l|}{ Interaction } \\
\hline $\mathrm{S}_{1} \mathrm{~F}_{1}$ & $100.37 \mathrm{de}$ & $100.60 \mathrm{c}-\mathrm{f}$ & $100.48 \mathrm{gh}$ & 198.54 ef & $198.78 \mathrm{e}-\mathrm{i}$ & $198.66 \mathrm{~d}-\mathrm{h}$ \\
\hline $\mathrm{S}_{1} \mathrm{~F}_{2}$ & $103.05 \mathrm{~b}-\mathrm{e}$ & $102.99 \mathrm{~b}-\mathrm{d}$ & $103.02 \mathrm{c}-\mathrm{g}$ & 200.90 c-e & $201.10 \mathrm{c}-\mathrm{g}$ & $201.00 \mathrm{c}-\mathrm{f}$ \\
\hline $\mathrm{S}_{1} \mathrm{~F}_{3}$ & 105.20 a-e & $105.33 \mathrm{a}-\mathrm{c}$ & $105.26 \mathrm{a}-\mathrm{g}$ & $205.91 \mathrm{a}-\mathrm{c}$ & $205.97 \mathrm{a}-\mathrm{d}$ & $205.94 \mathrm{a}-\mathrm{c}$ \\
\hline $\mathrm{S}_{1} \mathrm{~F}_{4}$ & $101.83 \mathrm{~b}-\mathrm{d}$ & $102.12 \mathrm{~b}-\mathrm{f}$ & $101.98 \mathrm{e}-\mathrm{h}$ & $199.33 \mathrm{~d}-\mathrm{f}$ & 200.20 d-h & 199.77 d-f \\
\hline $\mathrm{S}_{1} \mathrm{~F}_{5}$ & $104.80 \mathrm{a}-\mathrm{d}$ & $104.96 \mathrm{a}-\mathrm{c}$ & $104.88 \mathrm{a}-\mathrm{g}$ & 204.69 b-d & 205.07 a-e & $204.88 \mathrm{a}-\mathrm{d}$ \\
\hline $\mathrm{S}_{1} \mathrm{~F}_{6}$ & $109.22 \mathrm{a}$ & $106.57 \mathrm{a}-\mathrm{c}$ & $107.89 \mathrm{a}-\mathrm{c}$ & $207.64 \mathrm{ab}$ & $207.93 \mathrm{ab}$ & $207.79 \mathrm{ab}$ \\
\hline $\mathrm{S}_{1} \mathbf{F}_{7}$ & $94.45 \mathrm{f}$ & $96.17 \mathrm{f}$ & $95.31 \mathrm{i}$ & $192.05 \mathrm{~g}$ & $194.20 \mathrm{hi}$ & $193.13 \mathrm{gh}$ \\
\hline $\mathbf{S}_{2} \mathbf{F}_{1}$ & $101.43 \mathrm{c}-\mathrm{e}$ & $101.53 \mathrm{~b}-\mathrm{f}$ & $101.48 \mathrm{gh}$ & $198.37 \mathrm{ef}$ & $198.43 \mathrm{f}-\mathrm{i}$ & $198.40 \mathrm{e}-\mathrm{h}$ \\
\hline $\mathbf{S}_{2} \mathrm{~F}_{2}$ & $104.26 \mathrm{a}-\mathrm{d}$ & $104.24 \mathrm{a}-\mathrm{c}$ & $104.25 \mathrm{a}-\mathrm{g}$ & 201.64 c-e & 201.66 b-f & $201.65 \mathrm{~b}-\mathrm{e}$ \\
\hline $\mathrm{S}_{2} \mathrm{~F}_{3}$ & $107.56 \mathrm{ab}$ & $107.41 \mathrm{ab}$ & $107.49 a-d$ & $209.53 \mathrm{ab}$ & $209.63 \mathrm{a}$ & $209.58 \mathrm{a}$ \\
\hline $\mathbf{S}_{2} \mathbf{F}_{4}$ & $102.40 \mathrm{~b}-\mathrm{e}$ & $102.42 \mathrm{~b}-\mathrm{e}$ & $102.41 \mathrm{~d}-\mathrm{h}$ & $199.01 \mathrm{~d}-\mathrm{f}$ & $199.01 \mathrm{e}-\mathrm{i}$ & $199.01 \mathrm{~d}-\mathrm{g}$ \\
\hline $\mathrm{S}_{2} \mathrm{~F}_{5}$ & $106.92 \mathrm{a}-\mathrm{c}$ & $107.17 \mathrm{ab}$ & $107.04 \mathrm{a}-\mathrm{e}$ & 205.90 a-c & 205.90 a-d & $205.90 \mathrm{a}-\mathrm{c}$ \\
\hline $\mathrm{S}_{2} \mathrm{~F}_{6}$ & $108.93 \mathrm{a}$ & 109.89 a & $109.41 \mathrm{a}$ & $211.04 \mathrm{a}$ & $211.04 \mathrm{a}$ & $211.04 \mathrm{a}$ \\
\hline $\mathbf{S}_{2} \mathbf{F}_{7}$ & 97.50 ef & $97.33 \mathrm{~d}-\mathrm{f}$ & $97.42 \mathrm{hi}$ & $194.81 \mathrm{fg}$ & $194.81 \mathrm{~g}-\mathrm{i}$ & $194.81 \mathrm{f}-\mathrm{h}$ \\
\hline $\mathrm{S}_{3} \mathrm{~F}_{1}$ & $100.32 \mathrm{de}$ & $100.53 \mathrm{c}-\mathrm{f}$ & $100.43 \mathrm{gh}$ & 199.22 d-f & $199.41 \mathrm{e}-\mathrm{i}$ & $199.31 \mathrm{~d}-\mathrm{f}$ \\
\hline $\mathrm{S}_{3} \mathrm{~F}_{2}$ & $103.64 \mathrm{a}-\mathrm{d}$ & $103.86 \mathrm{a}-\mathrm{c}$ & $103.75 \mathrm{~b}-\mathrm{g}$ & 201.66 c-e & 201.85 b-f & 201.75 b-e \\
\hline $\mathrm{S}_{3} \mathrm{~F}_{3}$ & $109.15 \mathrm{a}$ & $107.08 \mathrm{ab}$ & $108.12 \mathrm{a}-\mathrm{c}$ & $208.31 \mathrm{ab}$ & $208.50 \mathrm{a}$ & 208.40 a \\
\hline $\mathrm{S}_{3} \mathbf{F}_{4}$ & $101.55 \mathrm{c}-\mathrm{e}$ & 101.76 b-f & $101.66 \mathrm{f}-\mathrm{h}$ & 199.13 d-f & $199.32 \mathrm{e}-\mathrm{i}$ & $199.23 \mathrm{~d}-\mathrm{f}$ \\
\hline $\mathrm{S}_{3} \mathrm{~F}_{5}$ & $106.64 \mathrm{a}-\mathrm{c}$ & $106.93 \mathrm{ab}$ & $106.78 \mathrm{a}-\mathrm{f}$ & $206.66 \mathrm{a}-\mathrm{c}$ & $206.85 \mathrm{a}-\mathrm{c}$ & $206.75 \mathrm{a}-\mathrm{c}$ \\
\hline $\mathrm{S}_{3} \mathrm{~F}_{6}$ & $107.44 \mathrm{ab}$ & $109.30 \mathrm{a}$ & $108.37 \mathrm{ab}$ & $210.95 \mathrm{a}$ & $210.71 \mathrm{a}$ & $210.83 \mathrm{a}$ \\
\hline $\mathbf{S}_{3} \mathbf{F}_{7}$ & $94.53 \mathrm{f}$ & 96.47 ef & $95.50 \mathrm{i}$ & $192.57 \mathrm{~g}$ & $193.20 \mathrm{i}$ & $192.89 \mathrm{~h}$ \\
\hline S.Em. \pm & 4.90 & 1.79 & 1.56 & 1.80 & 1.96 & \\
\hline
\end{tabular}

Means followed by the same letter (s) within a column are not significantly differed by DMRT $(\mathrm{P}=0.05)$

Initial grain Fe: 92.50 ppm in 2015 and 95.42 ppm in 2016 
Table.3 Zinc uptake by grain and fodder of sweet corn as influenced by agronomic fortification with zinc and iron

\begin{tabular}{|c|c|c|c|c|c|c|}
\hline \multirow[t]{2}{*}{ Treatments } & \multicolumn{3}{|c|}{ Zinc uptake by grain $\left(\mathrm{g} \mathrm{ha}^{-1}\right)$} & \multicolumn{3}{|c|}{ Zinc uptake by fodder $\left(\mathrm{g} \mathrm{ha}^{-1}\right)$} \\
\hline & 2015-16 & 2016-17 & Pooled & 2015-16 & 2016-17 & Pooled \\
\hline \multicolumn{7}{|l|}{ Main plot - Soil application } \\
\hline $\mathrm{S}_{1}-\mathrm{ZnSO}_{4} \& \mathrm{FeSO}_{4} @ 10 \mathrm{~kg} \mathrm{each} \mathrm{ha}^{-1}$ (No fortification) & $160.13 b$ & $171.43 \mathrm{~b}$ & $165.78 \mathrm{~b}$ & $331.17 \mathrm{~b}$ & $361.78 \mathrm{~b}$ & $346.48 \mathrm{~b}$ \\
\hline $\mathrm{S}_{2}-\mathrm{ZnSO}_{4} \& \mathrm{FeSO}_{4} @ 10 \mathrm{~kg} \mathrm{each} \mathrm{ha}^{-1}$ (Fortified with VC at $250 \mathrm{~kg}$ ) & 180.99 a & $194.48 \mathrm{a}$ & $187.73 \mathrm{a}$ & $377.52 \mathrm{a}$ & $416.27 \mathrm{a}$ & 396.89 a \\
\hline $\mathrm{S}_{3}-\mathrm{ZnSO}_{4} \& \mathrm{FeSO}_{4} @ 10 \mathrm{~kg} \mathrm{each} \mathrm{ha}^{-1}$ (Fortified with FYM at $500 \mathrm{~kg}$ ) & $174.58 \mathrm{a}$ & $187.83 \mathrm{a}$ & $181.20 \mathrm{a}$ & $362.51 \mathrm{a}$ & $396.86 \mathrm{a}$ & $379.68 \mathrm{a}$ \\
\hline S.Em. \pm & 2.33 & 2.35 & 2.31 & 5.78 & 5.61 & 5.66 \\
\hline \multicolumn{7}{|l|}{ Sub-plot - Foliar application } \\
\hline $\mathrm{F}_{1}-\mathrm{ZnSO}_{4} \& \mathrm{FeSO}_{4} @ 0.5 \%$ each at $20 \mathrm{DAS}$ & $145.48 \mathrm{~cd}$ & $158.11 \mathrm{c}$ & $151.80 \mathrm{~cd}$ & 289.84 e & $321.88 \mathrm{e}$ & $305.86 \mathrm{f}$ \\
\hline $\mathrm{F}_{2}-\mathrm{ZnSO}_{4} \& \mathrm{FeSO}_{4} @ 0.5 \%$ each at $40 \mathrm{DAS}$ & $165.96 \mathrm{c}$ & $179.10 \mathrm{c}$ & $172.53 \mathrm{c}$ & $358.96 \mathrm{c}$ & $392.20 \mathrm{c}$ & $375.58 \mathrm{~d}$ \\
\hline $\mathrm{F}_{3}-\mathrm{ZnSO}_{4} \& \mathrm{FeSO}_{4} @ 0.5 \%$ each at 20 and $40 \mathrm{DAS}$ & $200.48 \mathrm{ab}$ & $215.98 \mathrm{ab}$ & $208.23 \mathrm{ab}$ & $426.71 \mathrm{ab}$ & $465.79 \mathrm{ab}$ & $446.25 \mathrm{~b}$ \\
\hline $\mathrm{F}_{4}-\mathrm{ZnSO}_{4} \& \mathrm{FeSO}_{4} @ 1.0 \%$ each at $20 \mathrm{DAS}$ & $161.17 \mathrm{c}$ & $174.60 \mathrm{c}$ & $167.88 \mathrm{c}$ & $324.47 \mathrm{~d}$ & $361.39 \mathrm{~d}$ & $342.93 \mathrm{e}$ \\
\hline $\mathrm{F}_{5}-\mathrm{ZnSO}_{4} \& \mathrm{FeSO}_{4} @ 1.0 \%$ each at $40 \mathrm{DAS}$ & $186.20 \mathrm{~b}$ & $200.94 \mathrm{~b}$ & $193.57 \mathrm{~b}$ & $403.29 \mathrm{~b}$ & $439.86 \mathrm{~b}$ & $421.58 \mathrm{c}$ \\
\hline $\mathrm{F}_{6}-\mathrm{ZnSO}_{4} \& \mathrm{FeSO}_{4} @ 1.0 \%$ each at 20 and $40 \mathrm{DAS}$ & $213.53 \mathrm{a}$ & $228.54 \mathrm{a}$ & $221.04 \mathrm{a}$ & $451.04 \mathrm{a}$ & $491.50 \mathrm{a}$ & $471.27 \mathrm{a}$ \\
\hline $\mathrm{F}_{7}$ - Control (No foliar spray) & $130.46 \mathrm{~d}$ & $134.79 \mathrm{~d}$ & $132.62 \mathrm{~d}$ & $245.13 \mathrm{f}$ & $268.85 \mathrm{f}$ & $256.99 \mathrm{~g}$ \\
\hline S.Em. \pm & 6.92 & 7.10 & 6.97 & 8.51 & 9.57 & 8.16 \\
\hline \multicolumn{7}{|l|}{ Interaction } \\
\hline $\mathbf{S}_{1} \mathbf{F}_{1}$ & $139.91 \mathrm{e}-\mathrm{g}$ & 148.47 e-h & 144.19 e-h & $273.72 \mathrm{j}-1$ & $302.49 \mathrm{jk}$ & $288.101-n$ \\
\hline $\mathrm{S}_{1} \mathrm{~F}_{2}$ & $157.81 \mathrm{c}-\mathrm{g}$ & $169.61 \mathrm{c}-\mathrm{g}$ & $163.71 \mathrm{c}-\mathrm{g}$ & $336.64 \mathrm{f}-\mathrm{i}$ & $369.77 \mathrm{f}-\mathrm{i}$ & $353.21 \mathrm{~h}-\mathrm{j}$ \\
\hline $\mathrm{S}_{1} \mathrm{~F}_{3}$ & $175.08 \mathrm{~b}-\mathrm{e}$ & $188.13 \mathrm{~b}-\mathrm{e}$ & $181.61 \mathrm{~b}-\mathrm{f}$ & $382.87 \mathrm{~d}-\mathrm{f}$ & $416.36 \mathrm{~d}-\mathrm{f}$ & $399.61 \mathrm{e}-\mathrm{g}$ \\
\hline $\mathbf{S}_{1} \mathbf{F}_{4}$ & $157.22 \mathrm{c}-\mathrm{g}$ & $169.48 \mathrm{c}-\mathrm{g}$ & $163.35 \mathrm{c}-\mathrm{g}$ & 299.99 h-j & $334.35 \mathrm{~h}-\mathrm{j}$ & $317.17 \mathrm{j}-\mathrm{m}$ \\
\hline $\mathrm{S}_{1} \mathrm{~F}_{5}$ & $176.41 \mathrm{~b}-\mathrm{e}$ & $189.52 \mathrm{~b}-\mathrm{d}$ & $182.97 \mathrm{~b}-\mathrm{e}$ & $375.00 \mathrm{e}-\mathrm{g}$ & 408.01 ef & $391.51 \mathrm{f}-\mathrm{h}$ \\
\hline $\mathrm{S}_{1} \mathrm{~F}_{6}$ & $195.30 \mathrm{a}-\mathrm{c}$ & $207.81 \mathrm{a}-\mathrm{d}$ & $201.56 \mathrm{a}-\mathrm{c}$ & $421.95 b-d$ & $451.91 \mathrm{~b}-\mathrm{e}$ & $436.93 \mathrm{c}-\mathrm{e}$ \\
\hline $\mathbf{S}_{1} \mathbf{F}_{7}$ & $119.16 \mathrm{~g}$ & $126.97 \mathrm{~h}$ & $123.06 \mathrm{~h}$ & 228.021 & 249.581 & 238.80 o \\
\hline $\mathbf{S}_{2} \mathbf{F}_{1}$ & $151.56 \mathrm{~d}-\mathrm{g}$ & $167.12 \mathrm{~d}-\mathrm{g}$ & $159.34 \mathrm{~d}-\mathrm{h}$ & $305.02 \mathrm{~h}-\mathrm{j}$ & $341.02 \mathrm{~g}-\mathrm{j}$ & 323.02 i-1 \\
\hline $\mathrm{S}_{2} \mathrm{~F}_{2}$ & $173.08 \mathrm{~b}-\mathrm{e}$ & $188.45 \mathrm{~b}-\mathrm{e}$ & 180.77 b-f & $383.70 \mathrm{~d}-\mathrm{f}$ & $417.23 \mathrm{~d}-\mathrm{f}$ & $400.47 \mathrm{e}-\mathrm{g}$ \\
\hline $\mathrm{S}_{2} \mathrm{~F}_{3}$ & $217.50 \mathrm{a}$ & $234.68 \mathrm{a}$ & $226.09 \mathrm{a}$ & $452.89 \mathrm{a}-\mathrm{c}$ & $498.64 \mathrm{ab}$ & 475.77 a-c \\
\hline $\mathbf{S}_{2} \mathbf{F}_{4}$ & $164.15 c-f$ & $179.01 \mathrm{c}-\mathrm{f}$ & $171.58 \mathrm{c}-\mathrm{g}$ & $344.00 \mathrm{f}-\mathrm{h}$ & $383.08 \mathrm{f}-\mathrm{h}$ & $363.54 \mathrm{~g}-\mathrm{i}$ \\
\hline $\mathrm{S}_{2} \mathrm{~F}_{5}$ & $194.34 \mathrm{a}-\mathrm{c}$ & $210.68 \mathrm{a}-\mathrm{c}$ & $202.51 \mathrm{a}-\mathrm{c}$ & $424.94 \mathrm{a}-\mathrm{d}$ & $467.73 \mathrm{a}-\mathrm{d}$ & $446.33 \mathrm{~b}-\mathrm{d}$ \\
\hline $\mathrm{S}_{2} \mathrm{~F}_{6}$ & $224.72 \mathrm{a}$ & $240.06 \mathrm{a}$ & $232.39 \mathrm{a}$ & $470.23 \mathrm{a}$ & $517.09 \mathrm{a}$ & $493.66 \mathrm{a}$ \\
\hline $\mathbf{S}_{2} \mathrm{~F}_{7}$ & $141.56 \mathrm{e}-\mathrm{g}$ & $141.35 \mathrm{f}-\mathrm{h}$ & $141.46 \mathrm{f}-\mathrm{h}$ & $261.83 \mathrm{j}-1$ & $289.10 \mathrm{j}-1$ & $275.47 \mathrm{~m}-\mathrm{o}$ \\
\hline $\mathrm{S}_{3} \mathrm{~F}_{1}$ & $144.98 \mathrm{e}-\mathrm{g}$ & 158.75 e-h & 151.86 e-h & 290.79 i-k & $322.13 \mathrm{ij}$ & $306.46 \mathrm{k}-\mathrm{m}$ \\
\hline $\mathbf{S}_{3} \mathbf{F}_{2}$ & $167.00 \mathrm{c}-\mathrm{f}$ & $179.23 \mathrm{c}-\mathrm{f}$ & $173.12 \mathrm{c}-\mathrm{g}$ & $356.53 \mathrm{fg}$ & $389.60 \mathrm{fg}$ & $373.07 \mathrm{gh}$ \\
\hline $\mathrm{S}_{3} \mathrm{~F}_{3}$ & $208.85 \mathrm{ab}$ & $225.14 \mathrm{ab}$ & $216.99 \mathrm{ab}$ & $444.38 \mathrm{a}-\mathrm{c}$ & $482.36 \mathrm{a}-\mathrm{c}$ & $463.37 \mathrm{a}-\mathrm{d}$ \\
\hline $\mathrm{S}_{3} \mathrm{~F}_{4}$ & $162.13 \mathrm{c}-\mathrm{f}$ & $175.31 \mathrm{c}-\mathrm{g}$ & $168.72 \mathrm{c}-\mathrm{g}$ & $329.43 \mathrm{~g}-\mathrm{i}$ & $366.72 \mathrm{f}-\mathrm{i}$ & 348.08 h-k \\
\hline $\mathrm{S}_{3} \mathrm{~F}_{5}$ & $187.84 \mathrm{ab}$ & $202.62 \mathrm{a}-\mathrm{d}$ & $195.23 \mathrm{a}-\mathrm{d}$ & $409.93 \mathrm{c}-\mathrm{e}$ & $443.86 \mathrm{c}-\mathrm{e}$ & $426.89 \mathrm{~d}-\mathrm{f}$ \\
\hline $\mathrm{S}_{3} \mathrm{~F}_{6}$ & $220.57 \mathrm{a}$ & $237.75 \mathrm{a}$ & $229.16 \mathrm{a}$ & $460.95 \mathrm{ab}$ & $505.51 \mathrm{a}$ & $483.23 \mathrm{ab}$ \\
\hline $\mathrm{S}_{3} \mathrm{~F}_{7}$ & $130.67 \mathrm{fg}$ & $136.04 \mathrm{gh}$ & $133.35 \mathrm{gh}$ & $245.54 \mathrm{kl}$ & $267.86 \mathrm{kl}$ & 256.70 no \\
\hline S.Em. \pm & 11.98 & 12.30 & 12.08 & 14.73 & 16.57 & 14.14 \\
\hline
\end{tabular}

Means followed by the same letter (s) within a column are not significantly differed by DMRT $(\mathrm{P}=0.05)$ 
Table.4 Iron uptake by grain and fodder of sweet corn as influenced by agronomic fortification with zinc and iron

\begin{tabular}{|c|c|c|c|c|c|c|}
\hline \multirow[t]{2}{*}{ Treatments } & \multicolumn{3}{|c|}{ Iron uptake by grain $\left(\mathrm{g} \mathrm{ha}^{-1}\right)$} & \multicolumn{3}{|c|}{ Iron uptake by fodder $\left(\mathrm{g} \mathrm{ha}^{-1}\right)$} \\
\hline & 2015-16 & 2016-17 & Pooled & 2015-16 & 2016-17 & Pooled \\
\hline \multicolumn{7}{|l|}{ Main plot - Soil application } \\
\hline $\mathrm{S}_{1}-\mathrm{ZnSO}_{4} \& \mathrm{FeSO}_{4} @ 10 \mathrm{~kg} \mathrm{each} \mathrm{ha}^{-1}$ (No fortification) & $531.46 \mathrm{~b}$ & $567.40 \mathrm{~b}$ & $549.43 \mathrm{~b}$ & $1611.43 \mathrm{~b}$ & $1762.35 \mathrm{~b}$ & $1686.89 \mathrm{~b}$ \\
\hline $\mathrm{S}_{2}-\mathrm{ZnSO}_{4} \& \mathrm{FeSO}_{4} @ 10 \mathrm{~kg} \mathrm{each} \mathrm{ha}^{-1}$ (Fortified with VC at $250 \mathrm{~kg}$ ) & 586.93 a & $631.03 \mathrm{a}$ & $608.98 \mathrm{a}$ & $1791.17 \mathrm{a}$ & $1960.94 \mathrm{a}$ & $1876.05 \mathrm{a}$ \\
\hline $\mathrm{S}_{3}-\mathrm{ZnSO}_{4} \& \mathrm{FeSO}_{4} @ 10 \mathrm{~kg}$ each ha $^{-1}$ (Fortified with FYM at $500 \mathrm{~kg}$ ) & $567.03 \mathrm{ab}$ & $610.75 \mathrm{a}$ & $588.89 \mathrm{ab}$ & $1730.86 \mathrm{a}$ & $1892.65 \mathrm{a}$ & $1811.75 \mathrm{a}$ \\
\hline S.Em. \pm & 10.43 & 9.69 & 10.05 & 24.07 & 23.02 & 23.08 \\
\hline \multicolumn{7}{|l|}{ Sub-plot - Foliar application } \\
\hline $\mathrm{F}_{1}-\mathrm{ZnSO}_{4} \& \mathrm{FeSO}_{4} @ 0.5 \%$ each at $20 \mathrm{DAS}$ & 500.35 ef & $542.04 \mathrm{c}$ & 521.19 e & $1479.39 \mathrm{e}$ & $1635.11 \mathrm{~d}$ & $1557.25 \mathrm{e}$ \\
\hline $\mathrm{F}_{2}-\mathrm{ZnSO}_{4} \& \mathrm{FeSO}_{4} @ 0.5 \%$ each at $40 \mathrm{DAS}$ & $562.68 \mathrm{~cd}$ & $606.85 \mathrm{~b}$ & $584.77 \mathrm{~cd}$ & $1717.44 \mathrm{c}$ & $1869.27 \mathrm{c}$ & $1793.35 \mathrm{c}$ \\
\hline $\mathrm{F}_{3}-\mathrm{ZnSO}_{4} \& \mathrm{FeSO}_{4} @ 0.5 \%$ each at 20 and $40 \mathrm{DAS}$ & $626.59 \mathrm{ab}$ & $668.46 \mathrm{a}$ & $647.52 \mathrm{ab}$ & $1951.82 \mathrm{ab}$ & $2132.68 \mathrm{ab}$ & $2042.25 \mathrm{ab}$ \\
\hline $\mathrm{F}_{4}-\mathrm{ZnSO}_{4} \& \mathrm{FeSO}_{4} @ 1.0 \%$ each at $20 \mathrm{DAS}$ & $538.64 \mathrm{de}$ & $581.82 \mathrm{bc}$ & $560.23 \mathrm{de}$ & $1586.35 \mathrm{~d}$ & $1760.57 \mathrm{c}$ & $1673.46 \mathrm{~d}$ \\
\hline $\mathrm{F}_{5}-\mathrm{ZnSO}_{4} \& \mathrm{FeSO}_{4} @ 1.0 \%$ each at $40 \mathrm{DAS}$ & $593.96 \mathrm{bc}$ & $641.44 \mathrm{ab}$ & $617.70 \mathrm{bc}$ & $1872.22 \mathrm{~b}$ & 2033.86 b & $1953.04 \mathrm{~b}$ \\
\hline $\mathrm{F}_{6}-\mathrm{ZnSO}_{4} \& \mathrm{FeSO}_{4} @ 1.0 \%$ each at 20 and $40 \mathrm{DAS}$ & $655.85 \mathrm{a}$ & $701.79 \mathrm{a}$ & $678.82 \mathrm{a}$ & $2032.46 \mathrm{a}$ & 2204.96 a & $2118.71 \mathrm{a}$ \\
\hline $\mathrm{F}_{7}$ - Control (No foliar spray) & $454.57 \mathrm{f}$ & $479.03 \mathrm{~d}$ & $466.80 \mathrm{f}$ & $1338.39 \mathrm{f}$ & $1467.40 \mathrm{e}$ & $1402.90 \mathrm{f}$ \\
\hline S.Em. \pm & 17.70 & 20.33 & 18.77 & 33.04 & 38.23 & 31.95 \\
\hline \multicolumn{7}{|l|}{ Interaction } \\
\hline $\mathbf{S}_{1} \mathbf{F}_{1}$ & $476.45 \mathrm{f}-\mathrm{h}$ & $504.21 \mathrm{e}-\mathrm{h}$ & $490.33 \mathrm{~g}-\mathrm{i}$ & $1402.96 \mathrm{i}-\mathrm{k}$ & $1546.39 \mathrm{k}-\mathrm{m}$ & $1474.67 \mathrm{i}-\mathrm{k}$ \\
\hline $\mathrm{S}_{1} \mathrm{~F}_{2}$ & $538.91 \mathrm{~d}-\mathrm{g}$ & $576.70 \mathrm{c}-\mathrm{h}$ & $557.80 \mathrm{~d}-\mathrm{h}$ & $1620.91 \mathrm{f}-\mathrm{h}$ & $1776.94 \mathrm{~g}-\mathrm{j}$ & $1698.92 \mathrm{f}-\mathrm{h}$ \\
\hline $\mathbf{S}_{1} \mathbf{F}_{3}$ & $571.35 \mathrm{~b}-\mathrm{f}$ & $611.55 \mathrm{~b}-\mathrm{e}$ & $591.45 \mathrm{~b}-\mathrm{g}$ & $1816.98 \mathrm{c}-\mathrm{e}$ & $1979.42 \mathrm{~d}-\mathrm{g}$ & $1898.20 \mathrm{c}-\mathrm{e}$ \\
\hline $\mathrm{S}_{1} \mathbf{F}_{4}$ & $516.27 \mathrm{~d}-\mathrm{h}$ & $557.54 \mathrm{~d}-\mathrm{h}$ & $536.90 \mathrm{e}-\mathrm{i}$ & $1500.19 \mathrm{~h}-\mathrm{j}$ & $1672.27 \mathrm{i}-1$ & $1586.23 \mathrm{hi}$ \\
\hline $\mathrm{S}_{1} \mathrm{~F}_{5}$ & $573.09 \mathrm{~b}-\mathrm{f}$ & $614.61 \mathrm{~b}-\mathrm{e}$ & $593.85 \mathrm{~b}-\mathrm{g}$ & $1774.11 \mathrm{~d}-\mathrm{f}$ & 1926.40 e-h & $1850.26 \mathrm{~d}-\mathrm{f}$ \\
\hline $\mathrm{S}_{1} \mathrm{~F}_{6}$ & $618.37 \mathrm{a}-\mathrm{d}$ & $641.85 \mathrm{a}-\mathrm{d}$ & $630.11 \mathrm{a}-\mathrm{f}$ & $1902.92 \mathrm{~b}-\mathrm{d}$ & 2057.82 b-f & 1980.37 b-d \\
\hline $\mathrm{S}_{1} \mathrm{~F}_{7}$ & $425.76 \mathrm{~h}$ & $465.35 \mathrm{~h}$ & $445.55 \mathrm{i}$ & $1261.94 \mathrm{k}$ & $1377.19 \mathrm{~m}$ & $1319.57 \mathrm{k}$ \\
\hline $\mathrm{S}_{2} \mathrm{~F}_{1}$ & $527.47 \mathrm{~d}-\mathrm{g}$ & $578.25 \mathrm{c}-\mathrm{h}$ & $552.86 \mathrm{e}-\mathrm{h}$ & $1543.21 \mathrm{~g}-\mathrm{i}$ & $1712.30 \mathrm{~h}-\mathrm{k}$ & $1627.76 \mathrm{~g}-\mathrm{i}$ \\
\hline $\mathrm{S}_{2} \mathrm{~F}_{2}$ & $582.60 \mathrm{~b}-\mathrm{e}$ & $630.71 \mathrm{a}-\mathrm{d}$ & $606.66 \mathrm{~b}-\mathrm{f}$ & $1820.16 \mathrm{c}-\mathrm{e}$ & $1966.18 \mathrm{~d}-\mathrm{g}$ & $1893.17 \mathrm{c}-\mathrm{e}$ \\
\hline $\mathrm{S}_{2} \mathrm{~F}_{3}$ & $658.40 \mathrm{a}-\mathrm{c}$ & $708.86 \mathrm{ab}$ & $683.63 \mathrm{a}-\mathrm{c}$ & $2043.41 \mathrm{ab}$ & 2239.36 a-d & $2141.39 \mathrm{a}-\mathrm{c}$ \\
\hline $\mathrm{S}_{2} \mathbf{F}_{4}$ & $555.89 \mathrm{c}-\mathrm{f}$ & 601.83 b-f & $578.86 \mathrm{c}-\mathrm{g}$ & $1653.52 \mathrm{e}-\mathrm{h}$ & $1828.13 \mathrm{~g}-\mathrm{j}$ & $1740.82 \mathrm{e}-\mathrm{h}$ \\
\hline $\mathrm{S}_{2} \mathrm{~F}_{5}$ & $607.81 \mathrm{a}-\mathrm{d}$ & $660.29 a-d$ & $634.05 \mathrm{a}-\mathrm{e}$ & $1948.03 \mathrm{a}-\mathrm{d}$ & $2129.58 \mathrm{a}-\mathrm{e}$ & $2038.80 \mathrm{a}-\mathrm{c}$ \\
\hline $\mathrm{S}_{2} \mathrm{~F}_{6}$ & $687.48 \mathrm{a}$ & $743.44 \mathrm{a}$ & $715.46 \mathrm{a}$ & $2108.18 \mathrm{a}$ & $2294.73 \mathrm{a}$ & $2201.46 \mathrm{a}$ \\
\hline $\mathrm{S}_{2} \mathrm{~F}_{7}$ & $488.86 \mathrm{e}-\mathrm{h}$ & $493.84 \mathrm{f}-\mathrm{h}$ & $491.35 \mathrm{~g}-\mathrm{i}$ & $1421.65 \mathrm{i}-\mathrm{k}$ & $1556.30 \mathrm{k}-\mathrm{m}$ & $1488.98 \mathrm{i}-\mathrm{k}$ \\
\hline $\mathrm{S}_{3} \mathrm{~F}_{1}$ & $497.12 \mathrm{e}-\mathrm{h}$ & 543.65 d-h & $520.38 \mathrm{f}-\mathrm{i}$ & 1491.99 h-j & $1646.64 \mathrm{j}-1$ & $1569.32 \mathrm{~h}-\mathrm{j}$ \\
\hline $\mathrm{S}_{3} \mathrm{~F}_{2}$ & $566.53 \mathrm{~b}-\mathrm{f}$ & $613.15 \mathrm{~b}-\mathrm{e}$ & $589.84 \mathrm{~b}-\mathrm{g}$ & $1711.24 \mathrm{e}-\mathrm{g}$ & $1864.69 \mathrm{f}-\mathrm{i}$ & $1787.97 \mathrm{e}-\mathrm{g}$ \\
\hline $\mathrm{S}_{3} \mathrm{~F}_{3}$ & $650.01 \mathrm{a}-\mathrm{c}$ & $684.96 \mathrm{a}-\mathrm{c}$ & $667.49 \mathrm{a}-\mathrm{d}$ & $1995.07 \mathrm{a}-\mathrm{c}$ & $2179.25 \mathrm{a}-\mathrm{d}$ & $2087.16 \mathrm{ab}$ \\
\hline $\mathrm{S}_{3} \mathrm{~F}_{4}$ & $543.76 \mathrm{~d}-\mathrm{g}$ & $586.09 \mathrm{c}-\mathrm{g}$ & $564.93 \mathrm{~d}-\mathrm{h}$ & $1605.33 \mathrm{f}-\mathrm{h}$ & $1781.31 \mathrm{~g}-\mathrm{j}$ & $1693.32 \mathrm{f}-\mathrm{h}$ \\
\hline $\mathrm{S}_{3} \mathrm{~F}_{5}$ & $601.00 \mathrm{a}-\mathrm{d}$ & $649.43 \mathrm{a}-\mathrm{d}$ & $625.21 \mathrm{a}-\mathrm{f}$ & $1894.53 \mathrm{~b}-\mathrm{d}$ & $2045.61 \mathrm{c}-\mathrm{f}$ & $1970.07 \mathrm{~b}-\mathrm{d}$ \\
\hline $\mathrm{S}_{3} \mathrm{~F}_{6}$ & $661.68 \mathrm{ab}$ & $720.08 \mathrm{ab}$ & $690.88 \mathrm{ab}$ & 2086.27 a & $2262.33 \mathrm{ab}$ & $2174.30 \mathrm{a}$ \\
\hline $\mathrm{S}_{3} \mathrm{~F}_{7}$ & $449.10 \mathrm{gh}$ & $477.89 \mathrm{gh}$ & $463.50 \mathrm{hi}$ & $1331.59 \mathrm{jk}$ & $1468.70 \mathrm{~lm}$ & $1400.14 \mathrm{jk}$ \\
\hline S.Em. + & 30.66 & 35.22 & 32.52 & 57.23 & 66.22 & 55.33 \\
\hline
\end{tabular}

Means followed by the same letter (s) within a column are not significantly differed by DMRT $(\mathrm{P}=0.05)$ 
This may also due to the faster decomposition of organic and inorganic sources of nutrients and increases in the available cationic micronutrient concentration in soil solution thereby increasing the uptake of these micronutrients by sweet corn. Similar results were reported by Debele et al., (2001).

\section{References}

Anonymous, 2000. World Health Organisation, Geneva, Switzerland.

Bouis, H. E., 2003. Micronutrient fortification of plants through plant breeding can it improve nutrition in man at low cost. Proc. Nutri. Sci., 62 (2): 403-411.

Cababallero, B., 2002. Global patterns of child health the role of nutrition. Ann. Nutri. Metab, 46: 3-7.

Cakmak, I., 2008. Enrichment of cereal grains with zinc agronomic or genetic biofortification. Plant Soil, 30 (2): 1-17.

Debele, T., Shakarappa, K., Sudhir and Sujith, G. M., 2001. Direct and interactive effects of enriched micronutrients farm yard manure and nitrogen levels on the productivity and nutrient uptake of maize. Karnataka J. Agric. Sci., 14 (4): 894-899.

Follett, R. H. and Lindsay, W. L., 1969. Changes in DTPA extractable zinc, iron, manganese and copper in soils following fertilization. Soil Sci. Soc. American Proceed, 35: 600-602.

Habib, M., 2009. Effect of foliar application of $\mathrm{Zn}$ and $\mathrm{Fe}$ on wheat yield and quality. African. J. Biotechnol., 8 (24): 6795-6798.

Nayak, S. C. and Panda, D., 2000. Urea adsorption in alluvial soils. J. Indian Soc. Soil Sci., 48 (3): 179-181.

Salgueiro, M. J., Zubillaga, M., Lysionek, A., Sarabia, M. I., Caro, R., Paoli, T., Hager, A., Weill, R. and Boccio, J., 2000. Zinc as an essential micronutrient, a review. Nutri. Res., 20: $737-755$.

\section{How to cite this article:}

Fakeerappa Arabhanvi and Hulihalli, U.K. 2018. Agronomic Fortification with Zinc and Iron to Enhancing Micronutrient Concentration in Sweet corn grain to ameliorate the Deficiency Symptoms in Human Beings. Int.J.Curr.Microbiol.App.Sci. 7(02): 333-340. doi: https://doi.org/10.20546/ijcmas.2018.702.044 